\title{
Reactivity and Microstructure in the Epoxidation of Polybutadiene ${ }^{\dagger}$
}

\author{
Yasuhiko Kurusu,* Yoshiro Masuyama, and Michiko MiYamoto \\ Department of Chemistry, Faculty of Science and Technology, Sophia University, \\ 7-1 Kioicho, Chiyoda-ku, Tokyo 102, Japan
}

(Received March 9, 1994)

\begin{abstract}
We investigated the epoxidation of polybutadiene (PB) by peroxy acid generated in situ. Four types of polybutadienes were epoxidized by formic acid-hydrogen peroxide system. The micro structure of each is as follows: (cis\%) PB-1(98), (cis: trans: vinyl(pendant)\%) PB-2 (9:85:6), PB-4(36:55:9), (cis: vinyl(pendant)\%)) PB-3(10:90). The reactivity of the double bond decreased in the following order; trans cis vinyl(pendant). The selectivity of epoxidation decreased as follows; cis vinyl(pendant) trans. The double bonds of PB-1 reacted quantitatively, and the selectivity of the formation of epoxide group was $74 \%$. The ring opening of epoxide group obtained from trans-double bond in PB-2 occurred quickly by reaction with formic acid. The reacticity of PB-3 was lower than that of PB-1, because of the less-substituted double bond. PB-4 showed the same reactivity as PB-1, but gelation was observed when $60 \%$ of the double bonds reacted. Thus, we could obtain the product with well-defined structure by reaction of PB with a clearly determined micro structure. This method (formic acid-hydrogen peroxide system) was effective for the functionalization of PB.
\end{abstract}

KEY WORDS Epoxidation / Polybutadiene / Microstructure / in situ Peroxy Acid / Hydrogen Peroxide / Formic Acid /

The chemical modification of polymers is a post-polymerization process. This method is used for the improvement and optimization of chemical and mechanical properties and for the introduction of desirable functional groups in polymers. To synthesize a specialty polymer with certain desirable functional groups, the polymerization of monomers with these desirable functional groups is used. However there are often a number of difficulties associated with the reaction to obtain polymers from these monomers; (1) difficulty in polymerization, (2) side reactions during polymerization, (3) difficulty in the preparation of such a desirable monomer, and (4) unfavorable reactivity ratios in the case of copolymerization. The chemical modification of polymers with unsaturated bonds thus offers a potentially useful alternative method for the intro- duction of desirable functional groups on the polymer chains. The reaction of a macromolecule generally becomes complex owing to effects of neighboring groups. The functional group of the molecule shows various reactivities and macromolecules may be converted to many types of products.

As noted in the former report, ${ }^{1}$ the epoxidation of the polyolefin copolymer by in situ peroxy acid proceeded quantitatively. The reaction process was well followed by IR spectral studies, and transformation of the functional groups was clearly analyzed. We wish to set forth the epoxidation of polybutadiene (PB) by this method to extend its scope. Here, we deal with a polymer reaction, for we had previously reported that in situ generation of peroxy acid under heterogeneous conditions was effective for the epoxidation of

\footnotetext{
${ }^{\dagger}$ Higher Functionalization of Polyolefin by Chemical Modification III. For part II, see ref 1.
}

* To whom correspondence should be addressed. 
polyolefin copolymer. ${ }^{2}$ The epoxidation of unsaturated polymers by the reaction with peroxy acid offers a potentially useful method for improving and optimizing the mechanical and chemical resistance properties of diene type polymers. The introduction of epoxy groups gives the polymers hydrophilic property. There are many related reports about epoxidation of PB or butadiene-co-polymers, with monoperoxyphthalic acid, ${ }^{3} t$-butyl hydroperoxide/phosphoric acid, molybdate catalyst, ${ }^{4}$ and hydrogen peroxide/phosphoric acid, tungstate catalyst $^{5}$ were used as oxidizing reagents. There are one report ${ }^{6}$ and two patents ${ }^{6}$ about epoxidation by in situ generated peroxy acid (hydrogen peroxide-organic acid system). In these reports, the introduction ratio of oxirane was $20 \sim 37 \%$, but the results did not show the distinction of reactivity among the micro structures in PB. Also, as the reaction conditions were different, these were not comparable to each other. In this report, we investigate the relation between the microstructure of $\mathrm{PB}$ and reaction conditions in order to synthesize a higher functional polymer.

\section{EXPERIMENTAL}

\section{Materials and Measurements}

Polybutadiene $(P B)$. PB-1 and PB-4 were purchased from Aidrich Chemical Company, Inc. PB-2 and PB-3 were donated from Japan Synthetic Rubber Co., Ltd. These PBs were purified prior to reaction by the following method; $10 \mathrm{wt} \%$ of PB in methylene chloride was stirred for $24 \mathrm{~h}$ at room temperature. The solution was poured into methanol. The precipitate obtained was dried under reduced pressure. The purified PB was preserved as a white, rubber-like material in a desiccator. The microstructure was measured from FT-IR spectrum of PB film by Morero's method ${ }^{8}$ (FT-IR spectroscopic technique). The content of epoxy group and ester group in the product was assayed as follows. 1,2-Epoxy octane, 1-octene, and methyl formate were mixed in an arbitrary ratio. The absorbance of each functional group was measured from IR spectra of the mixture described above. An absorption coefficient was calculated from the absorbance and the concentration of each component in the authentic mixture. The results are shown in Tables I and II. Absorption coefficients of cis, trans, and vinyl(pendant) group of PB have been determined by Morero's method. In this experiment, we used 1-octene as a model of a vinyl(pendant) group of PB. The normalization of the absorption coefficient of the double bond was carried out as follows: cis: $740 \mathrm{~cm}^{-1}=0.21$, trans: 970 $\mathrm{cm}^{-1}=0.87$, pendant: $911 \mathrm{~cm}^{-1}=1.00$. Absorbances $(\varepsilon)$ of double bond, epoxy and ester group at the corresponding wave numbers described above were determined from FT-IR spectra. From the equation $\varepsilon=k c l(k=$ absorption coefficient, $c=$ concentration, $l=$ film thickness), $c l$ was calculated. As $l$ is a constant in this measurement, the ratio of the concentration of each group was determined.

Epoxidation of $P B$. In a typical run, $0.1 \mathrm{~g}$ of PB $\left(M_{w}=2.0 \times 10^{6}, 1.9 \mathrm{mmol}\right.$ eq. calculated from double bond units) were dissolved in $10 \mathrm{ml}$ of methylene chloride in a $50 \mathrm{ml}$ threenecked glass flask with a magnetic stirrer. To the polymer solution, $1.4 \mathrm{ml}(0.04 \mathrm{~mol})$ of $99 \%$ formic acid and $2.6 \mathrm{ml}(0.04 \mathrm{~mol})$ of $35 \%$ hydrogen peroxide were added. The resulting two-phase reaction mixture was vigorously stirred for a predetermined time. After com-

Table I. Determination of absorption coefficients of double bonds, and epoxy and ester groups

\begin{tabular}{crrr}
\hline & \multicolumn{3}{c}{ Compound conc./absorption coefficient } \\
\cline { 2 - 4 } & 1-Octene & 1,2-Epoxyoctane & Ethyl formate \\
\hline 1 & $1 / 1$ & $0.252 / 0.709$ & $0.706 / 3.290$ \\
2 & $1 / 1$ & $0.582 / 0.573$ & $1.077 / 3.051$ \\
3 & $1 / 1$ & $0.666 / 0.584$ & $0.966 / 3.106$ \\
4 & $1 / 1$ & $0.570 / 0.527$ & $0.496 / 4.436$ \\
5 & $1 / 1$ & $0.400 / 0.536$ & $0.450 / 3.968$ \\
Mean value & $/ 1$ & $/ 0.586$ & $/ 3.571$ \\
\hline
\end{tabular}


Table II. Wave number of functional groups and the normalized absorption coefficients

\begin{tabular}{|c|c|c|c|}
\hline Group & Key band $/ \mathrm{cm}^{-1}$ & $\begin{array}{c}\text { Absorption coefficient, } k \\
\text { (normalized in this experiment) }\end{array}$ & $\begin{array}{l}\text { Absorption coefficient } \\
\text { (Morero's method) }\end{array}$ \\
\hline Double bond: $c i s$ & 740 & 0.21 & 0.573 \\
\hline trans & 970 & 0.87 & 2.33 \\
\hline vinyl (pendant) & 911 & 1.00 & 2.67 \\
\hline Epoxy & 830 & 0.59 & \\
\hline Ester & 1725 & 3.57 & \\
\hline
\end{tabular}

pletion of the reaction, the water and organic layer were washed with aqueous sodium hydrogencarbonate solution and water. The film of the product was produced from the separated organic layer by casting it on water, and then was dried at room temperature under vacuum for at least 24 hours. The film obtained was analyzed by the method above.

\section{RESULTS AND DISCUSSION}

\section{Microstructure and Epoxidation of $P B$}

PB was analyzed by the method in the experimental section. The microstructure (cis/ trans/pendant ratios) of commercial products corresponded to catalog values. The values of PB-1, PB-2, PB-3, and PB-4 are summarized in Table III.

The influence of the concentrations of formic acid and hydrogen peroxide was investigated in the epoxidation of PB-3. The results are shown in Table IV. Adding excess formic acid to hydrogen peroxide reduced the selectivity to the epoxy group but increased the conversion of double bonds. As the reaction of double bond with formic acid was not observed, formic acid promoted carbon-oxygen bond cleavage of epoxy groups. The cleavage reaction was attributable to the high conversion of the double bonds in PB. When the concentration of formic acid was equal to or less than that of hydrogen peroxide, the conversion of double bonds and the selectivity of the epoxidation showed high values.

The epoxidation of PB-1 and PB-3 was performed at various reaction temperatures.
Table III. Microstructures of polybutadienes

\begin{tabular}{crcr}
\hline & & & \\
& cis $/ \%$ & trans $/ \%$ & pendant $/ \%$ \\
PB-1 & 98 & - & - \\
PB-2 & 9 & 85 & 6 \\
PB-3 & 10 & - & 90 \\
PB-4 & 36 & 55 & 9 \\
\hline
\end{tabular}

The results are shown in Table V. At $50^{\circ} \mathrm{C}$, the selectivity of epoxidation was low. The reaction was slow at $0^{\circ} \mathrm{C}$. The most appropriate temperature for obtaining high yield and high selectivity of the epoxidation was room temperature for the reaction of PB-1 and PB-3. The epoxidation of PB-1 was carried out room temperature in several solvents. The results are shown in Table VI. The reactivity decreased in the following order: methylene chloride $>$ chloroform $\sim$ ethylene chloride $>$ toluene $\sim$ carbon tetrachloride. This order was due to the solubility of the solvents to water. It was explained as follows: Performic acid as oxidant was at first formed in water phase, and then was transferred from the water phase into the organic phase. If the solubility shown in Table VI corresponds to the solubility of performic acid to the solvent, we can assume in this case that the solubility relates to the reactivity to the $\mathrm{PB}$. As the reaction of polybutadiene occurs in an organic phase, reactivities summarised in Table VI are consistent with the solubilities estimated from the solubility of water. The solubilities and 
Table IV. The investigation of the epoxidation condition (PB-3) ${ }^{\mathbf{a}}$

\begin{tabular}{|c|c|c|c|c|c|c|}
\hline No. & $\mathrm{HCOOH} / \mathrm{mol}$ & $\mathrm{H}_{2} \mathrm{O}_{2} / \mathrm{mol}$ & Reaction time/h & Conversion $^{b} / \%$ & Selectivity $/ \%$ & Yield $^{\mathrm{d}} / \%$ \\
\hline 1 & 0.04 & 0.01 & 6 & 25 & 58 & 14 \\
\hline 2 & 0.04 & 0.02 & 6 & 27 & 65 & 18 \\
\hline 3 & 0.04 & 0.04 & 6 & 26 & 75 & 19 \\
\hline 4 & 0.04 & 0.06 & 6 & 17 & 81 & 14 \\
\hline 5 & 0.04 & 0.1 & 6 & 14 & 80 & 11 \\
\hline 6 & 0.04 & 0.01 & 24 & 37 & 35 & 13 \\
\hline 7 & 0.04 & 0.02 & 24 & 37 & 48 & 18 \\
\hline 8 & 0.04 & 0.04 & 24 & 30 & 65 & 20 \\
\hline 9 & 0.04 & 0.06 & 24 & 19 & 80 & 15 \\
\hline 10 & 0.04 & 0.1 & 24 & 25 & 84 & 21 \\
\hline 11 & 0.02 & 0.04 & 6 & 19 & 88 & 17 \\
\hline 12 & 0.04 & 0.04 & 6 & 26 & 75 & 19 \\
\hline 13 & 0.06 & 0.04 & 6 & 34 & 64 & 21 \\
\hline 14 & 0.1 & 0.04 & 6 & 35 & 52 & 18 \\
\hline 15 & 0.02 & 0.04 & 24 & 28 & 80 & 22 \\
\hline 16 & 0.04 & 0.04 & 24 & 30 & 65 & 20 \\
\hline 17 & 0.06 & 0.04 & 24 & 45 & 47 & 21 \\
\hline 18 & 0.1 & 0.04 & 24 & 50 & 31 & 16 \\
\hline
\end{tabular}

a Reaction conditions: PB-3 $(0.1) \mathrm{g}$, methylene chloride $(10 \mathrm{ml})$, room temp.

b Conversion of olefin.

c Selectivity of epoxide.

d Yield of epoxide.

reactivity are shown in Table VI.

Epoxidations of PB-1, -2, -3, and -4 were carried out under the same conditions as above. The results are shown in Table V. A mixture of equal molar amounts of hydrogen peroxide and formic acid was used. The molar quantity of the peroxy acid obtained from the mixture was ten times the molar amount of the double bond in PB-1. It was confirmed by a preliminary experiment that the conditions were most appropriate. An appropriate reaction time for acquiring high yield and high selectivity of the epoxidation was $4 \mathrm{~h}$. in the reaction of $\mathrm{PB}$. The double bond in PB-2 showed high reactivity; all double bonds reacted, and epoxy groups were found only in one hour reaction. The cleavage of epoxy groups in PB-2 were found in the more long time reaction. Trans double bonds should be consumed via the formation of epoxide, since the double bond did not react with formic acid. The difference of reactivity between PB-1 and PB-2 might come from the difference of conformation between trans epoxy PB-2 and cis epoxy PB-1. This was shown from a molecular model depicted by the simulated annealing method in molecular dynamics, as shown in Figure 1. Trans epoxy polymer was not a compact packing, compared with cis epoxy polymer, and thus the ring opening reaction of trans epoxy group might proceed first. In the case of low molecular compounds, the ring opening of cis-epoxide is generally easier than that of trans epoxide. ${ }^{9}$ The polybutadiene treated in this experiment showed the opposite result. This result may come from effects from neighboring groups or conformation of the polymer chain, though elucidation of the effect was impossible in this experiment. The reactivity of the vinyl group of PB-3 was the lowest among PBs, because of the low electron density of terminal double bonds. When the conversion of double bonds exceeded $60 \%$, PB-4, a mixture of cis and trans structures, caused gelation. This gelation came from the reaction of cis-epoxide with a 
Epoxidation of Polybutadiene

Table V. Epoxidation of various $\mathbf{P B s}^{\mathbf{a}}$

\begin{tabular}{|c|c|c|c|c|c|c|c|}
\hline No. & $\mathrm{PB}(0.1 \mathrm{~g})$ & $\mathrm{HCOOH} / \mathrm{mol}$ & Reaction temp $/{ }^{\circ} \mathbf{C}$ & Time/h & Conv. $\mathbf{b} / \%$ & Select. ${ }^{c} \%$ & Yield $^{\mathrm{d}} / \%$ \\
\hline 1 & \multirow{12}{*}{ PB-1 } & 0.02 & 0 & 4 & 18 & 89 & 16 \\
\hline 2 & & 0.04 & 0 & 4 & 33 & 92 & 31 \\
\hline 3 & & 0.1 & 0 & 4 & 28 & 89 & 25 \\
\hline 4 & & 0.02 & room temp & 4 & 30 & 85 & 25 \\
\hline 5 & & $0.01^{*}$ & room temp & 4 & 42 & 94 & 39 \\
\hline 6 & & $0.04 *$ & room temp & 1 & 30 & 95 & 28 \\
\hline 7 & & $0.04 *$ & room temp & 2 & 38 & 94 & 36 \\
\hline 8 & & $0.04^{*}$ & room temp & 4 & 50 & 79 & 39 \\
\hline 9 & & 0.04 & room temp & 4 & 43 & 78 & 34 \\
\hline 10 & & 0.02 & 50 & 4 & 38 & 20 & 7 \\
\hline 11 & & 0.04 & 50 & 4 & 33 & 0 & 0 \\
\hline 12 & & 0.1 & 50 & 4 & 44 & 0 & 0 \\
\hline 13 & \multirow{8}{*}{ PB-2 } & $0.01^{*}$ & room temp & 4 & 44 & 0 & 0 \\
\hline 14 & & $0.02 *$ & room temp & 4 & 85 & 0 & 0 \\
\hline 15 & & $0.04 *$ & room temp & 1 & 13 & 60 & 8 \\
\hline 16 & & $0.04^{*}$ & room temp & 2 & 100 & 0 & 0 \\
\hline 17 & & $0.04^{*}$ & room temp & 4 & 100 & 0 & 0 \\
\hline 18 & & $0.04^{*}$ & room temp & 6 & 100 & 0 & 0 \\
\hline 19 & & $0.06^{*}$ & room temp & 4 & 100 & 0 & 0 \\
\hline 20 & & $0.1^{*}$ & room temp & 4 & 100 & 0 & 0 \\
\hline 21 & \multirow{9}{*}{ PB-3 } & 0.02 & room temp & 24 & 17 & 69 & 11 \\
\hline 22 & & 0.04 & room temp & 24 & 24 & 61 & 14 \\
\hline 23 & & $0.04^{*}$ & room temp & 1 & 4 & 88 & 3 \\
\hline 24 & & $0.04^{*}$ & room temp & 2 & 7 & 79 & 5 \\
\hline 25 & & $0.04^{*}$ & room temp & 4 & 12 & 75 & 9 \\
\hline 26 & & 0.1 & room temp & 24 & 32 & 63 & 20 \\
\hline 27 & & 0.02 & 50 & 24 & 25 & 43 & 11 \\
\hline 28 & & 0.04 & 50 & 24 & 40 & 18 & 7 \\
\hline 29 & & 0.1 & 50 & 24 & 100 & 0 & 0 \\
\hline 30 & \multirow{5}{*}{ PB-4 } & 0.1 & room temp & 4 & 44 & 44 & 19 \\
\hline 31 & & 0.04 & room temp & 1 & 14 & 81 & 12 \\
\hline 32 & & 0.04 & room temp & 2 & 31 & 40 & 13 \\
\hline 33 & & 0.04 & room temp & 3 & 55 & 43 & 24 \\
\hline 34 & & 0.04 & room temp & 4 & 59 & 28 & 17 \\
\hline
\end{tabular}

* Methylene chloride $(10 \mathrm{ml})$.

a Reaction conditions: $\mathrm{PB}(0.1 \mathrm{~g}), 1,2$-dichloroethane $(10 \mathrm{ml}), \mathrm{HCOOH} / \mathrm{H}_{2} \mathrm{O}_{2}=1$ (mol ratio).

b Conversion of olefin.

c Selectivity of epoxide.

d Yield of epoxide.

hydroxy or formyloxy group generated by acid-cleavage of an easily decomposable trans epoxy group, as observed in the reaction of PB-2.

The reactivity of formic acid-hydrogen peroxide reagent to olefins in PBs decreased in the following order; trans $>$ cis $>$ pendant. The selectivity of epoxidation was as follows; cis pendant $\gg$ trans $\fallingdotseq 0$.

PB-3 showed low selectivity in contrast with PB-1. This might be the reason for the solubility difference between PB-1 and PB-3. The film of PB-3 swelled in the formic acid-hydrogen peroxide solution, but there was 
Table VI. Epoxidation of PB-1 in various solvens ${ }^{a}$

\begin{tabular}{|c|c|c|c|c|c|c|}
\hline No. & Solvent $/ 10 \mathrm{ml}$ & $\mathrm{HCOOH} / \mathrm{mol}$ & $\mathrm{H}_{2} \mathrm{O}_{2} / \mathrm{mol}$ & Conv. $\mathbf{b} \%$ & Select. ${ }^{c} / \%$ & Yield $^{\mathrm{d}} / \%$ \\
\hline \multirow{2}{*}{1} & $\mathrm{CH}_{2} \mathrm{Cl}_{2}$ & 0.04 & 0.04 & 50 & 79 & 39 \\
\hline & $(22.5)^{\mathrm{e}}$ & 0.1 & 0.1 & 51 & 75 & 39 \\
\hline \multirow{2}{*}{2} & $\mathrm{CH}_{2} \mathrm{ClCH}_{2} \mathrm{Cl}$ & 0.04 & 0.04 & 33 & 98 & 32 \\
\hline & $(20.1)^{\mathrm{e}}$ & 0.1 & 0.1 & 44 & 98 & 43 \\
\hline \multirow{2}{*}{3} & $\mathrm{CHCl}_{3}$ & 0.04 & 0.04 & 21 & 93 & 20 \\
\hline & $(12.0)^{\mathrm{e}}$ & 0.1 & 0.1 & 9 & 100 & 9 \\
\hline \multirow{2}{*}{4} & $\mathrm{CCl}_{4}$ & 0.04 & 0.04 & 40 & 90 & 36 \\
\hline & $(2.0)^{\mathrm{e}}$ & 0.1 & 0.1 & 41 & 89 & 36 \\
\hline \multirow[b]{2}{*}{5} & $\mathrm{C}_{6} \mathrm{H}_{5} \mathrm{CH}_{3}$ & 0.04 & 0.04 & 4 & 92 & 4 \\
\hline & $(3.9)^{\mathrm{e}}$ & 0.1 & 0.1 & 16 & 96 & 15 \\
\hline
\end{tabular}

a Reaction conditions: PB- $1(0.1 \mathrm{~g}), 4 \mathrm{~h}$, room temp.

b Conversion of olefin.

c Selectivity of epoxide.

d Yield of epoxide.

e Solubility of water in the solvent $\left(\mathrm{H}_{2} \mathrm{O} \mathrm{mg} /\right.$ solvent $(10 \mathrm{ml})$. (The value was calculated from "Youzai Handbook (Solvent Handbook)," T. Asahara, N. Tokura, M. Okawara, J. Kumanotani, and M. Senoo, Ed., Kodan-sha Scientific, Tokyo, 1976.)

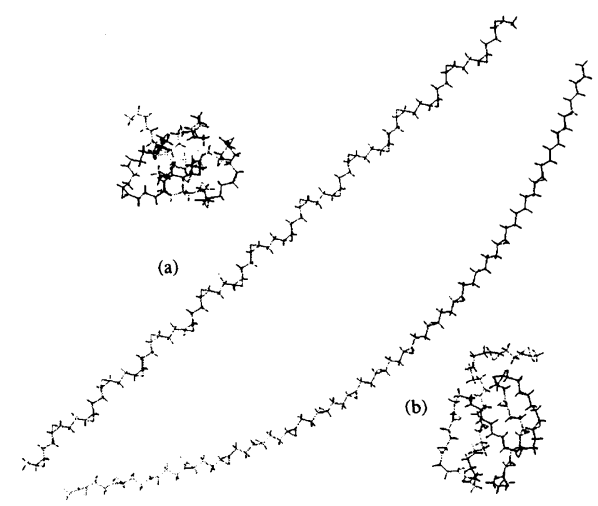

Figure 1. The conformation of cis-epoxide PB (a) and trans-epoxide PB drawed by a molecular dynamics method.

no swelling in the film of PB-1. Therefore, the selectivity of the epoxidation of PB-3 was low because of the easy cleavage of the epoxy group.

\section{CONCLUSION}

The best conditions for the epoxidation of
PB by performic acid generated in situ was the use of ten times for the double bond. The use of equimolar contents of hydrogen peroxide and formic acid was also essential for the best result. The epoxidation of cis double bonds showed high selectivity and high yield. The epoxidation of cis double bonds of PB was faster than that of terminal double bonds because of the difference of electron density on the double bonds. Trans double bonds in PB showed highest reactivity, and caused epoxidation followed by ring opening reaction to form hydroxy and formylate groups. When trans double bonds were present in polybutadiene, the epoxidation of polybutadiene was very difficult because of the easy ring opening reaction of the trans epoxy group with formic acid to form hydroxy and formyloxy groups. The reaction between these and the epoxy groups caused gelation. The epoxidation of trans double bond in PB would thus appear but not applicable because of the fast formation of hydroxy and formate groups through ring opening of the trans epoxy groups. 
Acknowledgment. The author is greatly indebted to Mr. Masato Takahashi in Dainippon Ink and Chemicals Inc. (Central Research Laboratories), for drawing the molecular structures of cis-epoxide and transepoxide of PB based on molecular dynamics.

\section{REFERENCES}

1. Y. Kurusu, Y. Masuyama, K. Yanagi, H. Morinaga, S. Yamamoto, and M. Nakanishi, Bull. Chem. Soc. Jpn., 66, 673 (1993).

2. Y. Kurusu, Y. Masuyama, T. Kato, K. Ohsawa, H. Morinaga, S. Yamamoto, and M. Nakanishi, Nippon Kagaku Kaishi (J. Chem. Soc. Jpn., Chem. Ind. Chem.), 840 (1992).

3. W.-K. Huang, G.-H. Hsiue, and W.-H. How, J.
Polym. Sci., A, 26, 1867 (1988); W.-K. Huang and G.-H. Hsiue, ibid., $A$, 27, 279 (1989).

4. M. Gahagan, A. Iraqi, D. C. Cupertino, R. K. Mackie, and D. J. Cole-Hamilton, J. Chem. Soc., Chem. Commun., 1688 (1989); A. Iraqi and D. J. Cole-Hamilton, J. Mater. Chem., 2, 183 (1992).

5. X. Jian and A. S. Hay, J. Polym. Sci., C, 28, 285 (1990); X. Jian and A. S. Hay, J. Polym. Sci., A, 29, 547 (1991); ibid., 1183 (1991).

6. M. Arnold and T. Langner, Plaste und Kautschuk mit Fachteil Anstrichstoffe, 37, 289 (1990).

7. T. Yasunaga, Y. Ito, and K. Fujita, Japan Patent, 56-45907 (1981); O. Hayashi, K. Kanda, and S. Matsumoto, Japan Patent, 61-148204 (1986).

8. D. Morero, A. Santanbrogio, L. Porri, and F. Clanpelli, Chim. e Ind., 41, 758 (1954).

9. G. H.Posner and D. Z. Rogers, J. Am. Chem. Soc., 99, 8208 (1977); J. Kagan, B. E. Firth, N. Y. Shih, and C. G. Boyajian, J. Org. Chem., 42, 343 (1977). 